\title{
Simultaneously effect of environment and sustained load on the degradation of aluminium bonded joint
}

\author{
Anwar SNR ${ }^{1,2,3}$, Crocombe $\mathrm{AD}^{2}$ \\ ${ }^{1}$ University of Mataram, NTB, Indonesia \\ ${ }^{2}$ University of Surrey, Guildford, GU2 7XH, UK \\ ${ }^{3}$ Ministry of Higher Education of Indonesia (DIKTI)
}

Email address:

rahmah_snra@yahoo.co.id (A. SNR), a.crocombe@surrey.ac.uk (Crocombe AD)

To cite this article:

Anwar SNR, Crocombe AD. Simultaneously Effect of Envinronment and Sustained Load on the Degradation of Aluminium Bonded Joint. International Journal of Materials Science and Applications. Vol. 2, No. 6, 2013, pp. 204-208. doi: 10.11648/j.ijmsa.20130206.16

\begin{abstract}
Bonded aluminium single lap joints have been exposed to a fully reversed ageing environment (immersion followed by withdrawal both at $50^{\circ} \mathrm{C}$ ) in both loaded and unloaded conditions with time dependent. Monitoring of these specimens and testing at key points on this reversed ageing environment has led to the stress rupture and moisture dependence of joint mechanical response included creep effect. Moisture cycling and stress influenced the tensile strength of the joints, with some apparent irreversible degradation. However, the effect of environmental exposure, time exposure, pre-treatment and constant stress was significant for the single lap joints damage.
\end{abstract}

Keywords: Adhesive Joint, Environment, Creep, Swelling, Damage

\section{Introduction}

Adhesive joints need to perform well under service conditions which include applied loads and exposure to hostile environments. The effect of simultaneous exposure to both mechanical stress and a fluctuating moisture environment is sometimes more severe than the each factor taken separately. This papers presents work investigating this combination of mechanical and environmental factors.

The moisture uptake can be analysed as single stage or dual stage Fickian diffusion [1, 2, 3]. Cyclic moisture diffusion tests have been carried out to characterise the moisture uptake of FM73-M OST [4, 5, 6]. Stress of the adhesive during diffusion can cause a more rapid absorption. [7-12]. Moisture diffusion causes a change of adhesive mechanical properties and the failure stress of adhesives reducing with increasing moisture [2, 3, 13, 14]. Creep deformation can occur in a loaded adhesive over a time with strain increasing with load, temperature, relative humidity $(\mathrm{RH})$ and time [3, 15]. and it is also it is possible that strength reductions will occur. Swelling occurs as an adhesive absorbs moisture and has been found to be uniquely linear with moisture content, even though depend on the different environments [2-3].

The experimental results limited on the situation of aircraft structures which are subjected environment loads (relative humidity, temperature) and sustained load simultaneously.

\section{Experimental Methods}

Various test procedures were employed to obtain the physical and mechanical properties of the adhesive FM73. Experiments were conducted on bulk adhesives and adhesively bonded single lap joints (SLJ) under static loading and a range of conditions (dry ; wet unloaded at 50 ${ }^{\circ} \mathrm{C}$ in deionised \& rain water ; wet loaded by constant stress in deionised and rain water at $50{ }^{\circ} \mathrm{C}$ and redry of its conditions).

\subsection{Specimen Manufacture}

Bulk adhesives and adhesively bonded aluminium joints were used in the experimental work. Bulk adhesives of 1 $\mathrm{mm}$ thickness were manufactured from nine layers of Cytec FM73 (double side tacky, polyester knit carrier) at a temperature of $120^{\circ} \mathrm{C}$. To investigate any surface effects on moisture uptake the surface of some of the bulk adhesive specimens were lightly roughened using medium grade abrasive paper.

The joints tested in thos research were made of aluminium alloy (2024-T3) substrates were bonded with Cytec FM73 to manufacture the SLJs. The dimensions of the joints are 
illustrated in Fig. 1. Aluminium 2024-T3 is an aluminium alloy is typically used in high strength applications. The aluminium surfaces were etched with chromic acid and anodised with phosphoric acid and then BR127 primer was applied to further enhance environmental resistance and durability. Specimens were exposed to deionised and rain water at $50{ }^{\circ} \mathrm{C}$ for 3 months and 6 months.
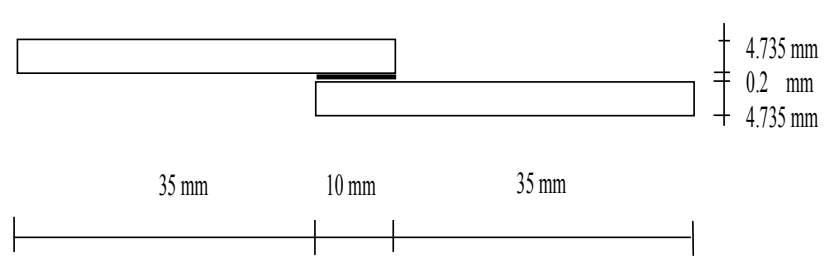

Figure 1. Single Lap Joint Specimens (width $3 \mathrm{~mm}$ )

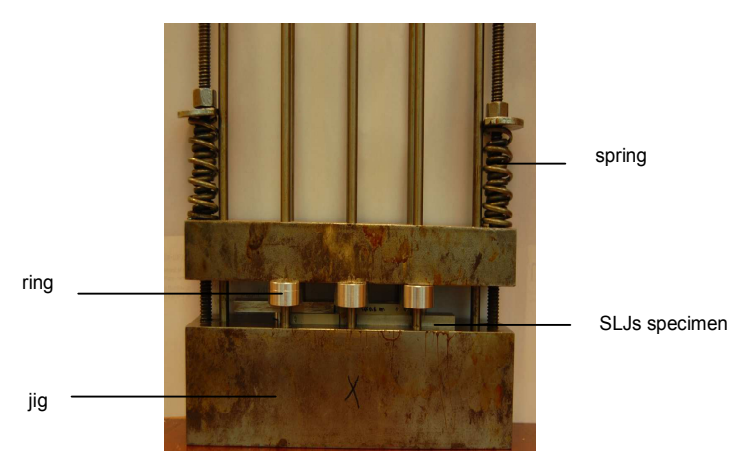

Figure 2. The jig used for the joints manufacturing

The aluminium was subject to chromic acid etching (CAE) and phosphoric acid anodising (PAA) followed with an application of $\mathrm{BR} \circledast 127$ corrosion inhibiting primer.

A jig was used to control the position of the aluminium (substrates), the thickness of bond line and the appropriate pressure. Two layers of adhesive sheet were used in the overlap area. The substrates, adhesive layers and the spacers were pressed in the jig. Then, the specimens were placed in the oven to heat up in 30 minutes to $120^{\circ} \mathrm{C}$ and were held for 60 minutes. After curing process ( cool down gradually overnight), the joints were kept in the desicators until testing.

\subsection{Combined Ageing and Loading Rig for the Bulk Adhesive and Adhesive Joint}

A test rig was designed incorporating springs to load the bulk adhesive and adhesive joint during immersion. Bulk adhesive specimens were immersed in deionised water in both unloaded and loaded $\left(25 \%\right.$ of the ultimate static load $\left(P_{u}\right)$ of the equivalent specimen) states until saturation followed by withdrawal until dry, both at $50{ }^{\circ} \mathrm{C}$. The single lap joints were immersed for 3 and 6 months in deionised and rain water, at $50{ }^{\circ} \mathrm{C}$ in both unloaded and loaded $\left(12 \% P_{u}\right)$ states and 6 months at the same environment by loaded $17.5 \% P_{u}$.

\subsection{Testing}

Gravimetric, swelling (measured using a digital micrometer), creep (determined through spring displacement in the rig) and quasi-static testing (on an
Instron test machine) were carried out on the bulk adhesive samples. Mechanical testing to measure the residual strength of the joints was also undertaken on an Instron test machine.

Tensile tests were carried out on single lap joints for all conditions (dry ; wet unloaded at $50{ }^{\circ} \mathrm{C}$ in deionised \& rain water ; wet loaded by constant stress in deionised and rain water at $50{ }^{\circ} \mathrm{C}$ and redry of its conditions) at $0.1 \mathrm{~mm} / \mathrm{min}$ of displacement rate

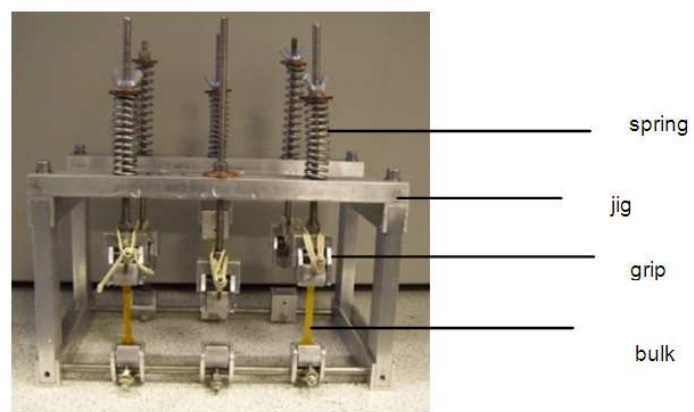

Figure 3. Jig with dogbone specimens loaded at $25 \%$ of their static failure load

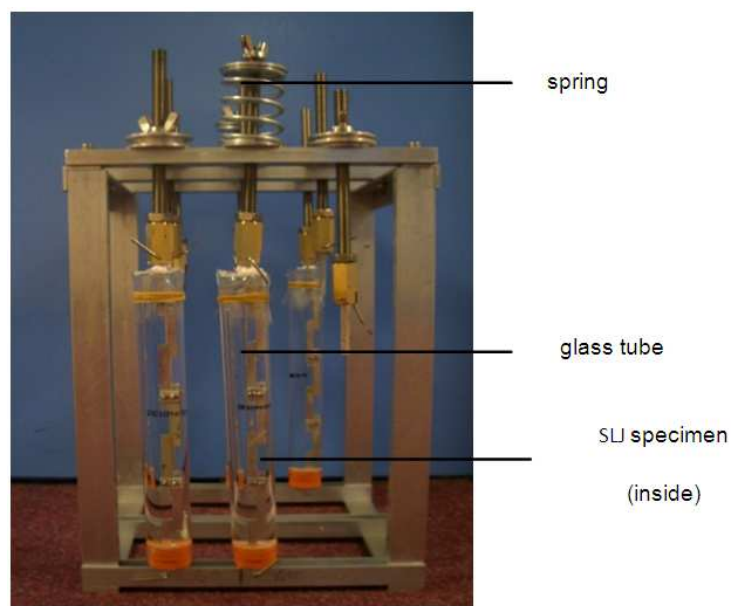

Figure 4. Jig for applying tensile load to Single Lap Joint during immersion

\section{Results and Discussion}

\subsection{Bulk Adhesive Testing}

The data measured from the bulk adhesive specimens consist of moisture (adsorption and desorption), swelling, creep and static stress-strain response. From the adhesive joint only static response was measured.

\subsubsection{Adsorption and Desorption}

Moisture uptake in the bulk adhesive specimens was aproximated as dual stage Fickian diffusion, which consists of two single Fickian processes. Desorption was fitted uing single stage Fickian diffusion. The experimental data was fitted to diffusion equations and the coefficients of diffusion obtained. A numerical iterative method was used to optimise the fit between the experimental data and the dual stage model. Fig. 5 until Fig. 8 show the final fit for moisture adsoption and desorption. 


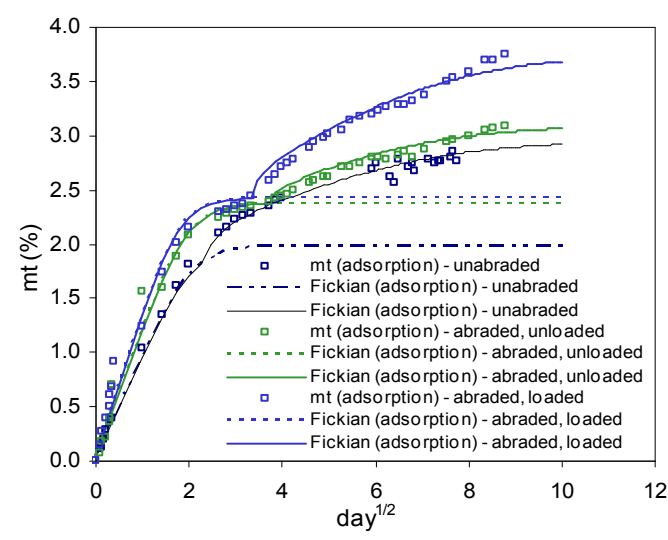

Figure 5. Absorption in FM73 bulk adhesive of unabraded, abraded unloaded (NL) and abraded loaded (L) surface for $1 \mathrm{~mm}$ thickness in deionised water

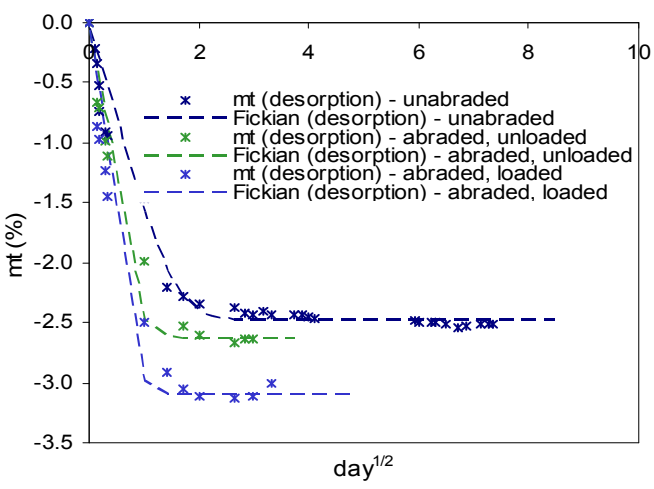

Figure 6. Desorption (b,d) in FM73 bulk adhesive of unabraded, abraded unloaded (NL) and abraded loaded (L) surface for $1 \mathrm{~mm}$ thickness in deionised water

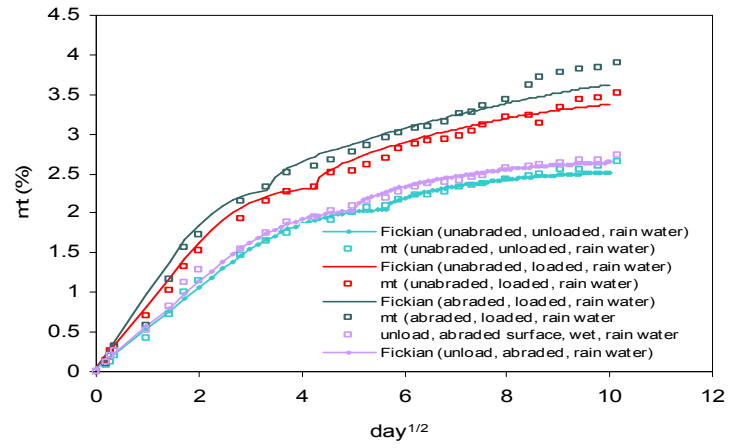

Figure 7. Absorption in FM73 bulk adhesive of unabraded, abraded unloaded $(N L)$ and abraded loaded (L) surface for $1 \mathrm{~mm}$ thickness rain water

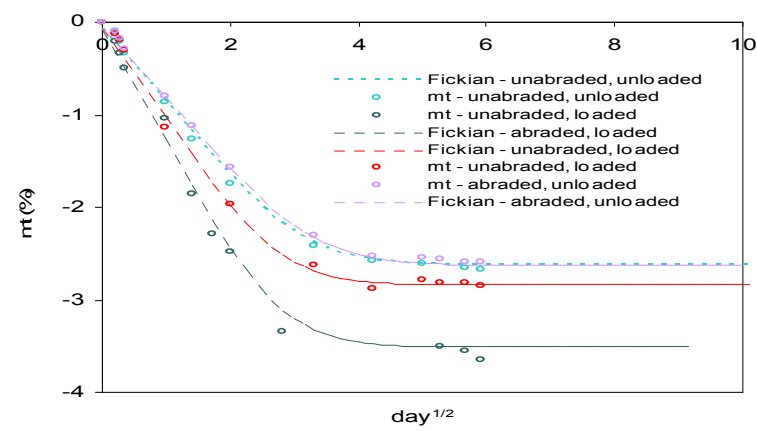

Figure 8. Desorption in FM73 bulk adhesive of unabraded, abraded unloaded (NL) and abraded loaded (L) surface for $1 \mathrm{~mm}$ thickness rain water

The diffusion adsorption desorption coefficients at different conditions exposed at $50{ }^{\circ} \mathrm{C}$ in deionised water and rain water can be found in Table 1 and Table 2 .

Table 1. Fickian diffusion coefficients of $1 \mathrm{~mm}$ FM73 ST thick adhesive samples (in deionised water)

\begin{tabular}{|c|c|c|c|c|c|}
\hline \multirow{2}{*}{ Condition } & \multirow{2}{*}{$\begin{array}{l}\text { Coefficient } \\
D_{1}\left(\mathrm{~m}^{2} / \mathrm{s}\right)\end{array}$} & \multirow{2}{*}{$\begin{array}{l}\text { Coefficient } \\
\mathrm{D}_{2}\left(\mathrm{~m}^{2} / \mathrm{s}\right)\end{array}$} & \multicolumn{3}{|c|}{ Saturated moisture content } \\
\hline & & & $\mathbf{M}_{1 \sim}(\%)$ & $\mathbf{M}_{2 \sim}(\mathrm{t} \%)$ & $\mathbf{M}_{\infty}(\%)$ \\
\hline \multicolumn{6}{|l|}{ Absorption : } \\
\hline No-abraded surface of FM73 & $5.21 .10^{-13}$ & $4.5010^{-14}$ & $2 . .36$ & 0.95 & 2.95 \\
\hline Abraded surface of FM73 no-loaded & $5.90 .10^{-13}$ & 4.28. $10^{-13}$ & 2.37 & 0.72 & 3.06 \\
\hline $\begin{array}{l}\text { Abraded surface of FM } 73 \text { loaded by } 25 \% \mathrm{P}_{\mathrm{u}} \\
\text { Desorption : }\end{array}$ & $7.18 .10^{-13}$ & $3.76 .10^{-13}$ & 2.44 & 1.31 & 3.75 \\
\hline No-abraded surface of FM73 & $3.01 .10^{-13}$ & & & & 2.49 \\
\hline Abraded surface of FM73 no-loaded & $6.94 \cdot 10^{-13}$ & & & & 2.64 \\
\hline Abraded surface of FM 73 loaded by $25 \% \mathrm{P}_{\mathrm{u}}$ & $3.76 .10^{-12}$ & & & & 3.10 \\
\hline
\end{tabular}

Table 2. Fickian diffusion coefficients of $1 \mathrm{~mm} F M 73$ BST thick adhesive samples (in rain water)

\begin{tabular}{|c|c|c|c|c|c|}
\hline \multirow{2}{*}{ Condition } & \multirow{2}{*}{$\begin{array}{l}\text { Coefficient } \\
D_{1}\left(\mathrm{~m}^{2} / \mathrm{s}\right)\end{array}$} & \multirow{2}{*}{$\begin{array}{l}\text { Coefficient } \\
D_{2}\left(\mathrm{~m}^{2} / \mathrm{s}\right)\end{array}$} & \multicolumn{3}{|c|}{ Saturated moisture content } \\
\hline & & & $M_{1 \sim}(\%)$ & $M_{2 \sim}(t \%)$ & $\mathbf{M}_{\infty}(\%)$ \\
\hline \multicolumn{6}{|l|}{ Absorption : } \\
\hline Un-abraded surface of FM73 & $1.50 .10^{-13}$ & $4.0010^{-14}$ & 2.05 & 0.60 & 1.50. $10^{-13}$ \\
\hline Abraded surface of FM73 unloaded & $1.74 \cdot 10^{-13}$ & $4.0510^{-14}$ & 2.08 & 0.65 & 1.74. $10^{-13}$ \\
\hline Un-abraded surface of FM 73 loaded by $25 \% \mathrm{P}_{\mathrm{u}}$ & $2.89 .10^{-13}$ & $2.90 .10^{-14}$ & 2.34 & 1.16 & 2.89. $10^{-13}$ \\
\hline $\begin{array}{l}\text { Abraded surface of FM73 loaded by } 25 \% \mathrm{P}_{\mathrm{u}} \\
\text { Desorption : }\end{array}$ & $3.70 .10^{-13}$ & $2.10 .10^{-14}$ & 2.33 & 1.57 & $3.70 .10^{-13}$ \\
\hline \multicolumn{6}{|l|}{ Un-abraded surface of FM73 } \\
\hline Abraded surface of FM73 unloaded & $3.01 \cdot 10^{-13}$ & & & & 2.49 \\
\hline Un-abraded surface of FM73 loaded by $25 \% \mathrm{P}_{\mathrm{u}}$ & $6.94 \cdot 10^{-13}$ & & & & 2.64 \\
\hline Abraded surface of FM73 loaded by $25 \% \mathrm{P}_{\mathrm{u}}$ & $3.76 .10^{-12}$ & & & & 3.10 \\
\hline
\end{tabular}




\subsubsection{Swelling and Creep}

The ageing sample thicknesses were measured periodically. The swelling was linear with moisture uptake (Fig. 7a), giving a coefficient of moisture expansion (CME) of $0.463 \%$ per $\% \mathrm{~m}_{\mathrm{t}}$ for un-abraded surface and $0.529 \%$ per $\% \mathrm{~m}_{\mathrm{t}}$ for abraded surface during diffusion in deionised water at $50{ }^{\circ} \mathrm{C}$. The $C M E$ during exposure in rain water at 50 $\mathrm{C}$ giving smaller value of $0.439 \%$ per $\% \mathrm{~m}_{\mathrm{t}}$ for un-abraded surface and $0.443 \%$ per $\% \mathrm{~m}_{\mathrm{t}}$ for abraded surface. Despite different rates of moisture uptake the swelling of the bulk adhesive with abraded and un-abraded surfaces were very similar. Long term creep in the bulk adhesive at $50{ }^{\circ} \mathrm{C}$ loaded at $25 \% P_{u}$ in deionised water were measured as creep-time curve (Fig $7 \mathrm{~b}$ ). It is possible to identify primary, secondary and maybe even the beginning of tertiary creep phases.

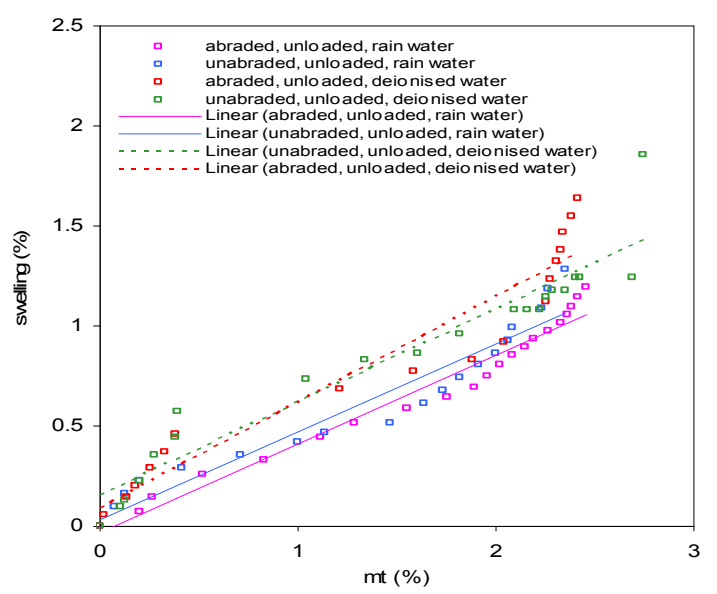

Figure 9. Swelling of FM73 bulk adhesive in deionised and rain water at 50 ${ }^{\circ} \mathrm{C}$

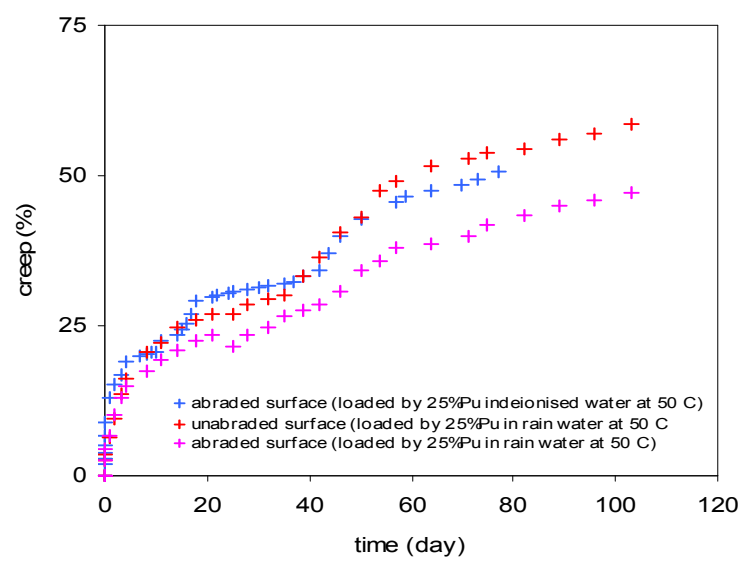

Figure 10. Creep of FM73 bulk adhesive in deionised and rain water at 50 ${ }^{o} \mathrm{C}$

\subsubsection{Static Response of the Bulk Adhesive and Adhesive Joint}

The static response of the bulk adhesive after exposure in deionised and rain water at $50{ }^{\circ} \mathrm{C}$ (Fig. 11) was obtained from mechanical tensile testing of bulk adhesive specimens at a rate of $0.1 \mathrm{~mm} / \mathrm{min}$ in the following states: a) dry, b) after immersion loaded by $25 \% P_{u}$, c) after immersion unloaded, d) dried following loaded by $25 \% P_{u}$ exposure and, e) dried following unloaded exposure in the deionised water. The residual tensile strength of bulk adhesive reduced from its unaged value by $8 \%, 28 \%, 2.4 \%$ and $4 \%$ for wet no load $(\mathrm{NL})$ and wet loaded (L), redried no load (NL) and redried loaded (L) respectively. While after exposure in rain water at $50{ }^{\circ} \mathrm{C}$, the residual strength decreased by $14 \%, 18 \%, 22 \%$ and $21 \%$ in the conditions : i) after immersion unloaded (un-abraded), ii) after immersion unloaded (abraded), iii) after immersion loaded by $25 \% P_{u}$ (abraded) and iv) after immersion loaded by $25 \% P_{u}$ (un-abraded). It seems that the full mechanical strength was not recovered on redrying.
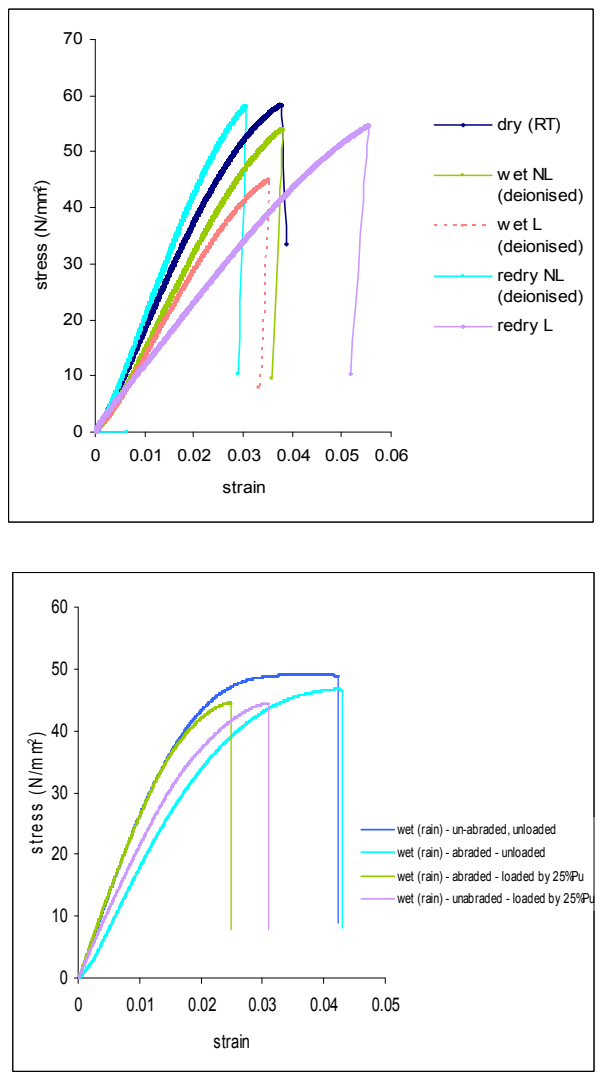

Figure 11. Tension test of bulk adhesive specimen

Joint strengths are shown in Fig 12. The strength of the single lap joints is determined by testing on the INSTRON 6025. The strength of the single lap joint when tested at 50 ${ }^{\circ} \mathrm{C}$ was reduced by about $23 \%$. The residual strength of single lap joint after absorption at $50^{\circ} \mathrm{C}$ in the deionised and rain water immersion; without load and under $12.5 \%$ ultimate load $\left(P_{u}\right)$ during immersion, are about : $1.63 \%$, $1.87 \%, 4.34 \%$ and $6.57 \%$ respectively. Comparing with other FM73-OST data, Mubashar (2009) has obtained the residual strength of absorption at $50{ }^{\circ} \mathrm{C}$ in deionised water for 8 weeks was $12.5 \%$. He also found $1.6 \%$ reduction following desorption. Liljedahl (2006) found the reduction was $46 \%$ after immersion for 16 weeks at the same 
conditions. This shows that time of exposure, environments and stress affected the joint strength. The residual strengths (stress and load) are detailed further in Table 3.

Table 3. Mechanical test of CAE Single Lap Joint at range conditions

\begin{tabular}{|c|c|c|c|c|c|}
\hline \multirow[t]{2}{*}{ No } & \multirow[t]{2}{*}{ Condition } & \multicolumn{2}{|c|}{ Mechanical Test } & \multicolumn{2}{|c|}{ Residual } \\
\hline & & Load $(\mathbb{N})$ & Stress $\left(\mathrm{N} / \mathrm{mm}^{2}\right)$ & Load (\%) & Stress $(\%)$ \\
\hline 1 & $D_{\text {ry }}(\mathrm{RT})$ & 1015991 & 33.866 & 0.00 & 0.00 \\
\hline 2 & $\operatorname{Dry}\left(50^{\circ} \mathrm{C}\right)$ & 782.310 & 26.089 & 23.00 & 22.96 \\
\hline 3 & 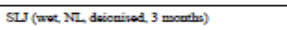 & 999.430 & 33.42 & 1.63 & 1.32 \\
\hline 4 & 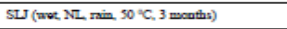 & 996.990 & 33.25 & 1.87 & 1.82 \\
\hline 5 & 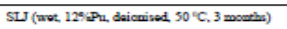 & 971.897 & 32.386 & 4.34 & 4.37 \\
\hline 6 & 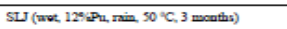 & 949.240 & 31.54 & 6.57 & 6.87 \\
\hline 7 & 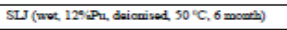 & 793.721 & 26.457 & 21.88 & 21.88 \\
\hline 8 & 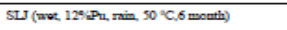 & 751.953 & 25.065 & 25.99 & 25.99 \\
\hline 9 & 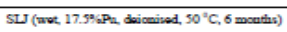 & 767.345 & 25578 & 24.47 & 24.47 \\
\hline 10 & 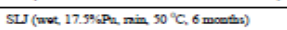 & 725.277 & 24.176 & 28.61 & 28.61 \\
\hline 11 & SIJ (raty, NL, deionised, $50^{\circ} \mathrm{C}, 1$ moestat) & 1003.996 & 33.467 & 1.18 & 1.18 \\
\hline 12 & $\mathrm{SLJ}$ (raty, NL, rain,, 50 "C, 1 maxthin) & 991.894 & 33.063 & 2.37 & 2.37 \\
\hline 13 & 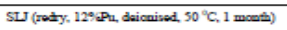 & 1000.714 & 33.357 & 1.50 & 1.50 \\
\hline 14 & 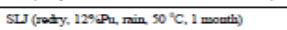 & 993.871 & 33.129 & 2.18 & 2.18 \\
\hline
\end{tabular}

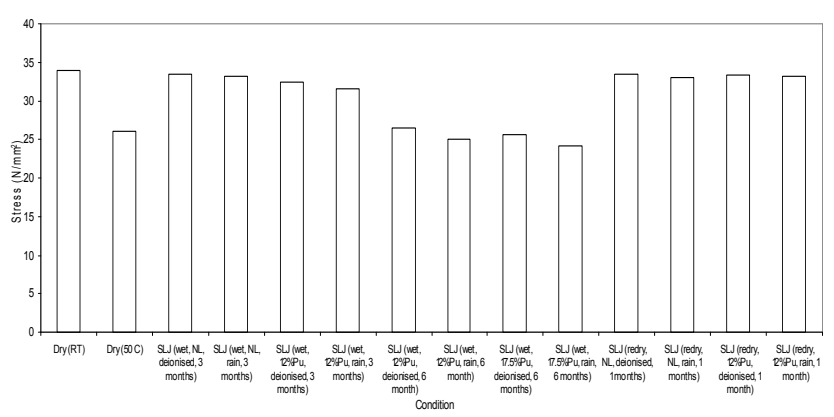

Figure 9. Residual load of single lap joint for a range of conditions

\section{Conclusions}

Based on the results of this research so far, it may be concluded that :

1. the rate diffusion with an abraded surface of bulk adhesive is faster than with a non-abraded surface.

2. the rate diffusion of bulk adhesive with loading during exposure is faster than without loading

3. the rate of desorption is faster than absorptions and not all moisture is removed.

4. the swelling of adhesive tend to be linear with the moisture uptake

5. significant creep extension occurred in the bulk adhesive exposed under loading with 3 stages ; primary, secondary and maybe even the beginning of tertiary creep phases.

6. full recovery of the bulk tensile properties was not recovered on redrying.

7. the joint performance was significantly degraded following exposure with time-dependent.

\section{Acknowledgments}

The author would like to thank the Ministry of Higher Education of Indonesia (DIKTI) for the funding of this project, University of Surrey UK for supplying the Laboratory of Composite and Mechanical.Laboratory

\section{References}

[1] Crank, J., 1975, "The Mathematic of Diffusion", $2^{\text {nd }}$ edition, Oxford University Press, London

[2] Loh, WK., Crocombe, AD., Wahab, MA., Watts, JF, Ashcroft, IA., "The Effect of Moisture On The Failure Locus And Fracture Energy Of An Epoxy-Steel Interface", Journal of Adhesion Science Technology, Vol.16 (11) : 1407-1429, 2002

[3] Liljedahl, CDM, Crocombe, AD., Wahab, MA., Ashcroft, IA., 2007, "Modelling The Environmental Degradation of Adhesively Bonded Aluminium And Composite Joints Using A CZM Approach", Elsevier International Journal of Adhesion \& Adhesives

[4] Mubashar, A., Aschroft, IA., Critchlow, GW., Crocombe, AD., 2009b, "Moisture AbsorptionDesorption Effects in Adhesive Joints", International Journals Adhesion and Adhesive 29 pp. 751-760

[5] Frigione M., Lettieri M., "Procedures Conditioning the Absorption/Desorption Behaviour of Cold-Cured Epoxy Resins", Journal of Polymet Science Vol. 46, p.1320-1336, 2008

[6] [6] McConnel BK., Pethrick RA., "Dielectric Studies of Water Absorption and Desorption in Epoxy Resins : Influence of Cure Process on Behaviour", Polymer International Journal, Vol. 57, p. 689-699, 2008

[7] Kim \& Brontman, 1971, 4th International Conference of Deformation, "Yield and Fracture of Polymers", Cambridge

[8] Gilliat O., Broutman LJ., ASTM STP No. 685, p.61-83, 1978

[9] Neumann S., Marom G., "Free-Volume Dependent Moisture Diffusion Under Stress in Composite Materials", Journal of Materials Science, Vol. 21, p. 26-30, 1986

[10] Kasturiarachchi KA and Pritchard G., "Water Absorption of Glass/Epoxy Laminates Under Bending Stresses", Journals of Composites Vol. 14, p. 244-250, 1983

[11] Knox, E.M. and Cowling, M.J., "A Rapid Durability Test Method for Adhesives", International Journal of Adhesion \& Adhesives. Vol. 20, p. 201-208, 2000.

[12] Roy S., Vengadassalam K., Wang Y, Park S and Liechti K.M., "Characterization and Modeling of Strain Assisted Diffusion in An Epoxy Adhesive Layer ",International Journal of Solids and Structures, Vol. 43, p. 27-52, 2006

[13] Jurf \& Vinson, 1985, Journal of Material Science, 20, 2979-2989

[14] Cabanelas, J.C., Prolongo S.G., Serrano B., Bravo J., Baselga, 2003, Journal of material processing technology, In press

[15] Adhesivestoolkit.com, Granta Park, Great Abington, Cambridge, 2010 